\title{
Study of Potential Application of Cyanobacteria Leptolyngbya Valderiana in Skin Cosmeceuticals
}

\author{
Shraddha Ratnaparkhe*, Devyani Mali, Bela Nabar \\ Department of Microbiology, Smt. C.H.M college, Ulhasnagar-3, Maharashtra, India-421003
}

\begin{abstract}
Skin is the outermost cover and first line of defense of the human body. Skin cosmeceuticals are therapeutic agents used to maintain flawless skin and prevent skin disorders. Considering the ill effects of synthetic active agents, natural metabolites are preferred for skincare. Cyanobacterium was known to produce various secondary metabolites with therapeutic potential. This study focuses on screening whole-cell extract of Leptolyngbya valderiana for skincare. Isolation and identification of cyanobacteria were made. The whole-cell extract of Leptolyngbya valderiana was studied for its metabolite profile, mainly for proteins, carbohydrate, and lipid content. Screening of whole-cell extract was carried out for its (2,2'-azino-bis (3-ethylbenzothiazoline-6-sulfonic acid)) (ABTS) radical scavenging ability and UV absorption potential. The antimicrobial effect of extracts was determined by agar cup and minimum inhibitory concentration (MIC) method using salicylic acid as a standard. Skin whitening potential was determined using tyrosinase inhibition assay. Leptolyngbya valderiana was isolated from the tree bark and selected for further study. The extract of Leptolyngbya valderiana was found to have $25.22 \%$ proteins, $36.65 \%$ carbohydrates and $35.97 \%$ lipids. The extract was found to have antioxidant potential with $96.55 \%$ ABTS radical scavenging potential. Antimicrobial potency of the extract against Staphylococcus aureus ATCC 6538, Propionibacterium acne MTCC 1351, Candida albicans ATCC 10231 was equivalent to salicylic acid. The test extract was found to inhibit the tyrosinase enzyme by $43.46 \%$. The test extract is rich in proteins, carbohydrates, and lipids. The extract has significant antioxidant potential, antimicrobial efficiency, ability to absorb ultraviolet B and ultraviolet A region, tyrosinase inhibition potential indicative photoprotective, and skin whitening potential. The isolate Leptolyngbya valderiana has a promising potential for skin cosmeceuticals.
\end{abstract}

Keywords: Antiacne, Applications, Leptolyngbya valderiana, Skin cosmeceuticals, Skin whitening, Tyrosinase.

\section{INTRODUCTION}

Skin is the outermost layer of the human body comprising $20 \%$ of the body's total surface area. This largest organ plays a significant role as a protective barrier and in the regulation of body temperature. It acts as a defensive agent against the invasion of foreign particles, microorganisms, and xenobiotic compounds. With aging, the skin becomes thin, loses fat, elasticity, and smoothness. Different dermatological conditions are observed based on certain factors like medicinal treatment, air pollution, heredity factors, stress, and UV rays, leading to melanoma or skin cancer. The common dermatological conditions are hyperpigmentation, freckles, age spots, uneven skin tone, melasma, rosacea, and skin aging, which is mainly caused by overexposure to UV. ${ }^{1}$

To maintain healthy skin, various skin care products and cosmetics are used. However, synthetic chemicals have their demerits. Sunscreen agents like titanium dioxide and zinc oxide are not preferred because of their problems related to photostability and cross-reactions. ${ }^{2}$ Many synthetic agents cause contact dermatitis, skin irritancy, phototoxicity, allergy, and sometimes skin cancer. Moreover, most of the products are expensive, and not scientifically tested and validated for the skincare claims mentioned on the product. Natural ingredients are potent, cheap, safe alternatives to synthetic agents. ${ }^{3}$ The cosmeceutical market is rapidly emerging in India due to green cosmetics, mainly ayurvedic preparations. As per the statistics of CII, Confederation of Indian Industries, the current 600 million US dollars market may increase by $15-20 \%{ }^{4}$ Therefore, natural ingredients are focused for their application study in various cosmeceuticals.

Over the past decades, much research has been done to apply cyanobacteria in biodiesel production, skin, and the pharmaceutical industry. ${ }^{5}$ The bioactive metabolites of cyanobacteria like high-quality proteins, essential amino acids, polyunsaturated amino acids,vitamins phenolics, flavonoids, lipids, and polysaccharides have various therapeutic applications. ${ }^{6,7}$

\section{Corresponding author:}

Shraddha Ratnaparkhe

Email: shraddha.rp1@gmail.com

Received: 21-07-2021 
Leptolyngbya, is a well-known genus of cyanobacteria that is widely spread in nature and observed in the fresh ecosystem as well as extreme environments. The genus is rich in secondary metabolites in response to stress conditions in the environment. Most of the bioactive metabolites are structurally novel but not much exploration is done in their application study. Therefore, this study focuses on the application of active metabolites of Leptolyngbya valderiana in skincare application.

\section{MATERIAL AND METHODS}

\subsection{Isolation and Biochemical Characterization of Algal Isolates}

The cyanobacterial isolate Leptolyngbya valderiana was isolated from specimen EB01 collected from tree barks of Eucalyptus globufrom Taloja region of Maharashtra. The isolate was identified microscopically based on morphology using monographs. Further confirmation was done using $16 \mathrm{~S}$ rRNA sequencing method using primers. Forward primer 8F with sequence 5'AGATTTGATCCTGGCTCAG 3'\& reverse primer 907R 3'CCGTCAATTCMTTTRAGTTT $5^{\prime}$ were used for the study. ${ }^{8}$

\subsection{Biochemical Characterization of Leptolyngbya valderiana}

The isolate was characterized for various metabolite content.

\subsubsection{Estimation of Intracellular Proteins}

Extraction of intracellular proteins from dry pellet was done using lysis buffer consisting of $100 \mathrm{nM}$ Tris (hydroxymethyl) aminomethane (Tris) buffer, $10 \mathrm{nM}$ Ethylene-diaminetetracetic acid (EDTA), 150 mM TritonX-100, $0.09 \mathrm{mM}$ phenylmethylsulfonyl fluoride (PMSF), $0.65 \mathrm{mM}$ Dithiothreitol (DTT). After centrifugation at $13500 \mathrm{rpm}$ for 20 minutes, the supernatant was collected for protein estimation by Folin-Lowry method. Bovine serum albumin $(1 \mathrm{mg} / \mathrm{mL})$ was used as a standard. To $0.2 \mathrm{~mL}$ test solution, $2 \mathrm{~mL}$ of alkaline $\mathrm{CuSO}_{4}$ reagent was added and incubated at room temperature for $10 \mathrm{~min}$. Then, $0.2 \mathrm{~mL}$ of $50 \%$ Folin-ciocalteu reagent was added. After incubation at room temperature for 30 minutes, absorbance was measured at $585 \mathrm{~nm}$ on Kanad Vidyut 0392 spectrophotometer. ${ }^{9}$

\subsubsection{Estimation of Carbohydrate}

Extraction of carbohydrates was done by treating the dry powder of Leptolyngbya valderiana with $250 \mu \mathrm{L}$ of concentrated sulfuric acid at $30^{\circ} \mathrm{C}$ for 1-hour. After the addition of $7 \mathrm{~mL}$ distilled water, heating was done in a boiling water bath for 10 minutes. After cooling, $400 \mu \mathrm{L}$ of $4 \%$ sulfuric acid was added in $100 \mu \mathrm{l}$ of test extract. The $4 \%$ sulfuric acid was used in blank and glucose $(50 \mathrm{mg} / \mathrm{mL})$ as standard. The tubes were heated in a boiling water bath for 10 min after adding $2 \mathrm{~mL}$ of Anthrone reagent. After cooling, absorbance was measured at $585 \mathrm{~nm}$ using kanad Vidyut 0392 spectrophotometer. ${ }^{9,10}$

\subsubsection{Estimation of Lipids}

Lipids were extracted with methanol: chloroform (1:2) with overnight incubation at $4^{\circ} \mathrm{C}$. After centrifugation, the supernatant was collected and mixed with $1.3 \mathrm{~mL}$ distilled water and $3 \mathrm{~mL}$ chloroform. The chloroform layer containing lipids was separated, dried completely, and treated with $100 \mu \mathrm{L}$ concentrated sulfuric acid at $100^{\circ} \mathrm{C}$ for 10min. After cooling, $2.5 \mathrm{~mL}$ of sulfovanillin (SPV) reagent was added and incubated at room temperature for $20 \mathrm{~min}$, absorbance was measured at $530 \mathrm{~nm}$ in Kanad Vidyut 0392 spectrophotometer. ${ }^{11}$

\subsection{Study of the Application of Whole-cell Extracts of the Cyanobacteria}

\subsubsection{Preparation of Whole-cell Extract}

Extraction of dry powder of Leptolyngbya valderiana was done using tetrahydrofuran and methanol (2:8) with overnight incubation at $4^{\circ} \mathrm{C}$. The supernatant was evaporated at room temperature for 18-24 hours, stored in the refrigerator at $4^{\circ} \mathrm{C}$ until further use.

Partial purification was done by modification of the method described by Rastogi et al. 2013. ${ }^{12}$ The partially purified extract of Leptolyngbya valderiana was prepared by treating the dry whole cell extract with water: chloroform in 6:1 ratio. The tubes were gently vortexed and then centrifuged at $13500 \mathrm{rpm}$. The aqueous layer containing MAA was separated after centrifugation and stored at $4^{\circ} \mathrm{C}$ until further use. ${ }^{13}$

\subsubsection{Determination of the Antioxidant Activity of Algal Extracts using ABTS Assay}

The ABTS (2,2'-Azino-bis (3-ethylbenzothiazioline6 -sulfonic acid) radical was prepared by mixing $2.45 \mathrm{nM}$ potassium persulfate and $7 \mathrm{mM}$ ABTS solution $(1 / 1 \mathrm{v} / \mathrm{v})$ and stabilized by incubation at room temperature for 16-20 hours. The absorbance of ABTS radical was adjusted to $0.7 \pm 0.05$ using $50 \%$ methanol. For the assay, the reaction mixture of $200 \mu \mathrm{L}$ of test extract in methanol and $1800 \mu \mathrm{L}$ of ABTS solution was incubated at room temperature for 6 min. Ascorbic acid was used as standard. The absorbance is measured at $700 \mathrm{nM}$ on Kanad Vidyut 0392 spectrophotometer. ${ }^{14}$ Percent inhibition was determined with the following formula:

$$
\text { Percent inhibition of ABTS }=\frac{(\text { Abs control }- \text { Abs test })}{\text { Abs control }} \times 100
$$

Where, Abs control = absorbance of the control and Abs test $=$ Absorbance of test 


\subsubsection{Screening of Whole-cell Extract of Cyano- bacteria for their UVA and UVB Absorption}

The whole-cell extracts as well as partially purified extracts of Leptolyngbya valderiana were studied spectroscopically for their photoprotection potential. Both the samples were scanned between $200 \mathrm{nM}$ to $400 \mathrm{nM}$ using UV spectrophotometer (Shimatzu UV 1800) to monitor absorption patterns in UVA, UVB region. ${ }^{15}$

\subsubsection{Screening of Whole Cell Extract of Cyanobacteria for Antimicrobial Activity Against Skin Pathogens}

The antimicrobial activity of whole-cell extract of Leptolyngbya valderiana in dimethyl sulfoxide (DMSO) was evaluated against three test organisms namely, Propionibacterium acnes MTCC 1951, Staphylococcus aureus ATCC 6538, and Candida albicans ATCC 10231 by agar cup method. ${ }^{16}$. The log phase test culture was seeded on Muller Hinton Agar plate using pour plate method. The wells were inoculated with $100 \mu \mathrm{l}$ of test culture. The plates with $S$. aureus and $P$. acnes were incubated at $32^{\circ} \mathrm{C}$ for 24 hours. in aerobic and anaerobic conditions, respectively. Salicylic acid was used as a standard.

\subsubsection{Determination of Minimum Inhibitory Concentration of Whole-Cell Extract of Leptolyngbya Valderiana}

The whole cell extract of Leptolyngbya valderiana was screened for its antimicrobial potency using a minimum inhibitory assay. The stock solution of test extract $(1 \mathrm{mg} /$ $\mathrm{ml}$ ) was diluted in 10\% DMSO to get a final concentration of $250 \mu \mathrm{L}, 500 \mu \mathrm{L}, 750 \mu \mathrm{l}$, and $1000 \mu \mathrm{L}$. To the $5 \mathrm{~mL}$ diluted sample, $100 \mu 1$ of 24 -hour old test culture was added. Then test samples inoculated with $S$. aureus and $P$. acne were incubated at $37^{\circ} \mathrm{C}$ for $24 \mathrm{hr}$. in aerobic and anaerobic conditions, respectively. The samples inoculated with C. albicans were incubated at $25^{\circ} \mathrm{C}$ for 48 hours. Salicylic acid was used as standard. ${ }^{9,16}$

\subsubsection{Screening of whole-cell extract of algae for inhibition of mushroom tyrosinase enzyme using L-tyrosine as substrate}

The reaction mixture consisted of $400 \mu \mathrm{L}$ of $5 \mathrm{nM}$ phosphate buffer, $200 \mu \mathrm{L}$ of test extract in DMSO, $200 \mu \mathrm{L}$ of tyrosinase enzyme (200 units $/ \mathrm{mL}$ ) and incubation was done at $37^{\circ} \mathrm{C}$ for 10 minutes. Kojic acid was used as standard. Absorbance was measured at $475 \mathrm{nM}$. Percent inhibition was calculated as follows. ${ }^{17,18}$

$$
\text { Percent inhibition of tyrosinase }=\frac{(\text { Abs control }- \text { Abs test })}{\text { Abs control }} \times 100
$$

Where, Abs control = absorbance of the control and Abs test $=$ Absorbance of test

\section{RESULTS}

\subsection{Isolation and Identification of Cyanobacteria}

The microscopic observation showed the morphology of test isolates as long thin filaments either straight or coiled forms and the filaments were surrounded by a very thin sheet as depicted in Figure 1. Based on morphology, the isolate was found to be from Leptolyngbya genus. Based on the results of 16s RNA sequencing analysis, the isolate was identified as Leptolyngbya valderiana.

\subsection{Biochemical Characterization of Leptolyngbya valderiana}

\subsubsection{Estimation of Proteins}

The estimation of proteins of Leptolyngbya valderiana was done using Folin-Lowry method. As indicated in Figure 2, the protein content was found to be $25.22 \pm 0.06 \%$. Cyanobacterial proteins are the source of essential amino acids. The peptides also provide nutrition to improve skin glow. The results are in congruence to the research work of Gharbia et al in 2019. They reported 10\% and 30\% protein content in two Leptolyngbya strains studied by them. The test isolate of Leptolyngbya valderiana is rich in protein content and has trivial application in the nutraceutical, cosmetic and pharmaceutical industries. ${ }^{19}$

\subsubsection{Estimation of carbohydrates}

The carbohydrate content in the isolate Leptolyngbya valderiana was $36.65 \pm 0.06 \%$ as depicted in Figure 2. Gharbia et al. 2019 reported the carbohydrate content of Leptolyngbya sp. in the range of $15-47 \%$. The high content of carbohydrates is associated with the mucilaginous sheath in Leptolyngbya valderiana. ${ }^{19}$ The carbohydrates help in skin lubrication thus keep the skin smooth and moist. They also act as viscosity boosters and thickening agents in skincare products.

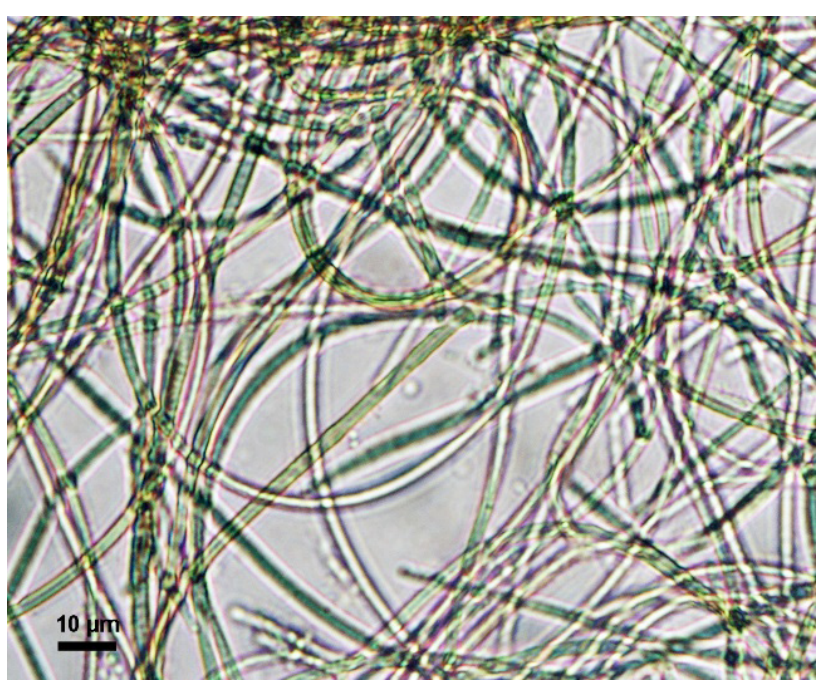

Figure 1: Microscopic image of Leptolyngbya valderiana under 40x 


\subsubsection{Estimation of Lipids:}

The lipid content of Leptolyngbya valderiana was found to be $35.97 \pm 0.47 \%$. They act as emollient. The lipid content was higher in this cyanobacteria compared to the average lipid content reported $5.85 \%$ in cyanobacteria from southwest Indian ocean lands. ${ }^{20}$ Lipids play an important role in skin nourishment and in maintaining the permeability barrier of the skin. The lipid content of Leptolyngbya valderiana was higher than the lipid content of Leptolyngbya strains reported in previous studies. ${ }^{21}$ The results are presented in Figure 2.

\subsection{Study of the Application of Whole-cell Extracts of the Cyanobacteria:}

\subsubsection{Estimation of Antioxidant Activity by ABTS Method}

The whole-cell methanolic extract of Leptolyngbya valderiana was assessed for its antioxidant potential as per ABTS assay. The extract was found to have $96.55 \pm 0.26 \%$ ABTS free radical scavenging capacity. The antioxidant potential of Leptolyngbya valderiana is greater than the standard used ascorbic acid.

\subsubsection{Screening of Whole-cell Extract of Cyano- bacteria for their UVA and UVB Absorption}

The whole-cell extract of Leptolyngbya valderiana was screened for absorption in UVA and UVB region. The maximum absorption was observed in the region of 320 $\mathrm{nm}$ to $340 \mathrm{~nm}$ (UVA) region as shown in Figure 3.

\subsubsection{Screening of whole-cell extract of} cyanobacteria for antimicrobial activity against skin pathogens

The antimicrobial potential of whole-cell extract of cyanobacteria was evaluated using agar cup method. The experiment was done in triplicate. The average zone of inhibition was found to be $34.17 \pm 0.29 \mathrm{~mm}, 34.33 \pm 1.04$, and $34.16 \pm 2.08 \mathrm{~mm}$ against Staphylococcus aureus ATCC 6538, Propionibacterium acenes MTCC 1351, and Candida albicans ATCC 10231, respectively.

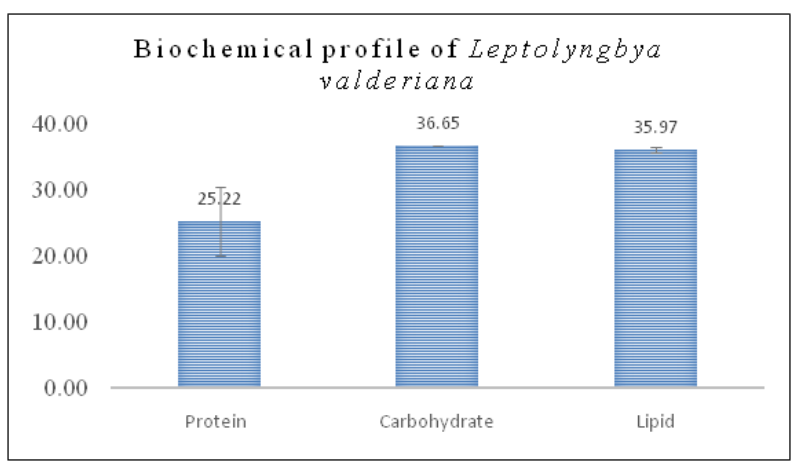

Figure 2: Biochemical profile of Leptolyngbya valderiana
The zone of inhibition of salicylic acid was observed as $41.67 \pm 0.58 \mathrm{~mm}, 41.33 \pm 0.76,41.33 \pm 1.04 \mathrm{~mm}$ against Staphylococcus aureus ATCC 6538, Propionibacterium acenes MTCC 1351, and Candida albicans ATCC 10231, respectively. Based on Kirby Bauer's chart, the extract was found to have antimicrobial efficacy against all three test organisms.

\subsubsection{Determination of Minimum Inhibitory Concentration of Whole-cell Extract of Leptolyngbya valderiana}

The minimum inhibitory concentration of wholecell extract of Leptolyngbya valderiana was found to be $500 \mu \mathrm{g} / \mathrm{mL}$ for all three test organisms. The MIC value is equivalent to the MIC value of salicylic acid, a antiacne agent used commercially. This indicates that whole-cell extracts of Leptolyngbya valderiana has antimicrobial potential against various skin pathogens similar to that of salicylic acid, as presented in Figure 4.

\subsubsection{Estimation of Tyrosinase Inhibition Assay using Tyrosine as Substrate}

The whole-cell DMSO extract of Leptolyngbya valderiana was assessed for its tyrosinase inhibition potential. The extract was found to have $43.46 \pm 0.25 \%$ tyrosinase inhibition. The IC50 value of whole cell extracts of DMSO extract of Leptolyngbya valderiana was $1161.98 \pm 7.54 \mu \mathrm{g} / \mathrm{mL}$.

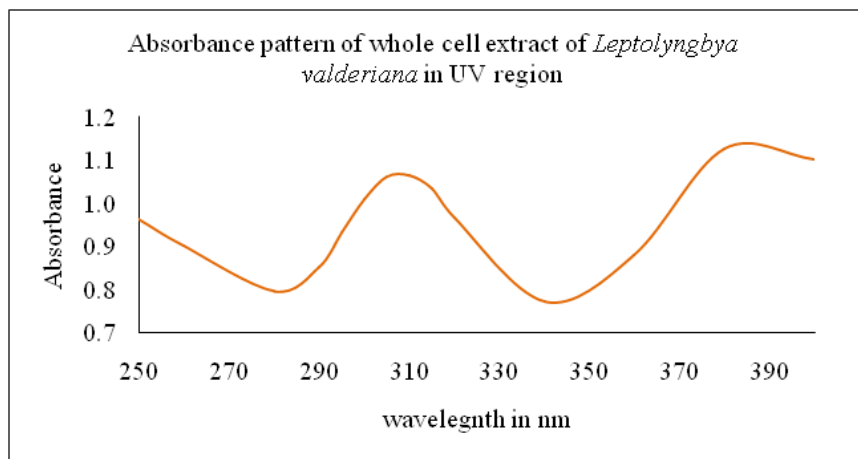

Figure 3: Screening of whole cell extract of Leptolyngbya valderiana for photoprotection using UV spectrophotometer

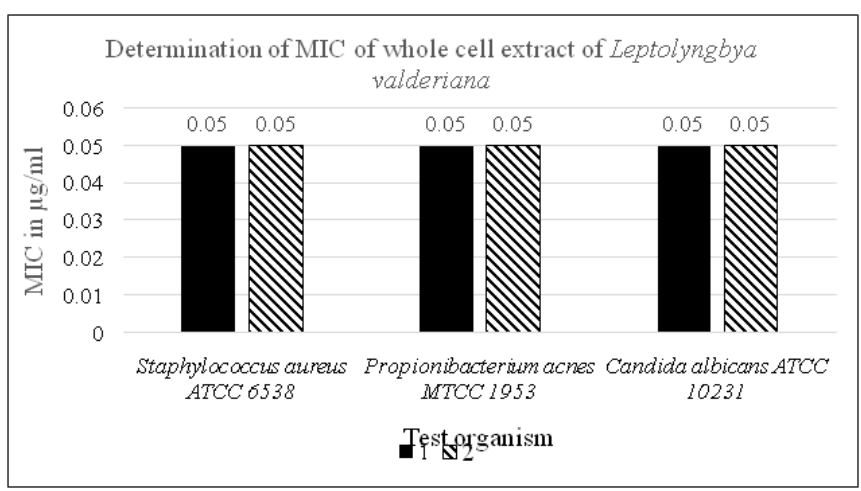

Figure 4: The minimum inhibitory concentration of whole cell extract of Leptolyngbya valderiana against various test organisms. 


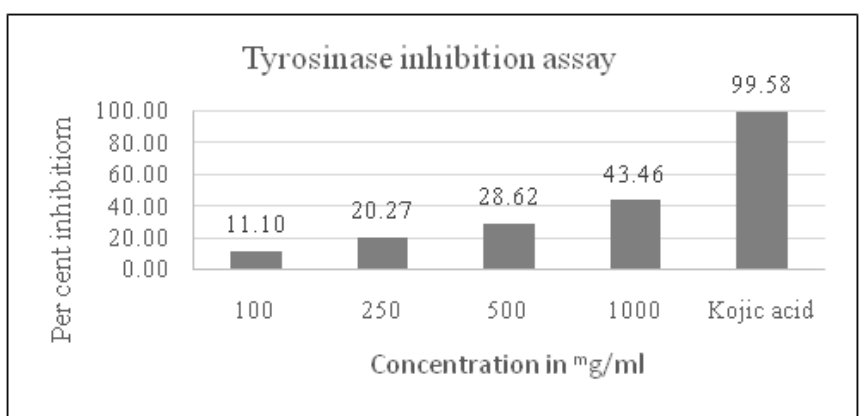

Figure 5: Tyrosinase inhibition assay of the whole cell DMSO extract of Leptolyngbya valderiana

Kojic acid showed $99.58 \%$ inhibition. The tyrosinase inhibition potential of a whole cell extract of Leptolyngbya valderiana is less than $50 \%$ potential of Kojic acid as indicated in Figure 5.

\section{DISCUSSION}

Skin disorders are mainly associated with various stressors like UV rays, bacterial infections. The UVA rays lead to tanning or hyperpigmented skin, suppression of immunological function, depletion of antioxidants, and premature aging. Whereas UVB rays lead to skin burn. ${ }^{22}$ The extract can absorb UV radiations mainly in UV. A region which can prevent photodamage of skin. Free radicals like hydrogen peroxide $\left(\mathrm{H}_{2} \mathrm{O}_{2}\right)$, hypochlorous acid $\left(\mathrm{HOCl}^{*}\right)$, hydroxyl radicals $(\mathrm{OH})$ generated in various biological reactions damage cell membranes, various proteins, fats, and nucleic acids in cells, thus resulting in various disorders, mainly pigmentation of the skin, wrinkle formation and aging. ${ }^{23}$ The whole extract of Leptolyngbya valderiana has significant antioxidant capacity. Therefore, the extract can combat free oxygen radicals preventing various disorders associated with ROS. The partially purified extract also showed a significant amount of UVA absorption, indicating richness in mycosporine-like amino acids.

The whole-cell extract of Leptolyngbya valderiana was also screened for antimicrobial potential. The extract was antimicrobial against all the three test organisms, namely Staphylococcus aureus ATCC 6538, Propionibacterium acne MTCC 1351, and Candida albicans ATCC 10231. The efficacy of whole-cell extract of Leptolyngbya valderiana is equivalent to salicylic acid, which is used as an antimicrobial agent, commercially available. The extract can protect the skin from microbial infections, especially acne. As mentioned in previous studies, the extract of Leptolyngbya valderiana was found to have skin whitening potential, which helps in reducing pigmentation and dark spots. ${ }^{9}$ The extract of Leptolyngbya valderiana was also rich in proteins, carbohydrates, and lipids. The proteins provide skin nutrition, and carbohydrates maintain skin lubrication. The lipids improve the permeability barrier of the skin and act as emollient agents.

\section{CONCLUSION}

The whole-cell extract has multiple applications in skin care products as an anti-acnes agent, antioxidant, photoprotective agent, skin emollient, skin moisturizer, and skin nourishment agent. The extract has multipurpose applications not only for skin protection but also for skincare formulation. Thus, it has a competitive advantage over synthetic chemicals.

\section{ACKNOWLEDGMENT}

We acknowledge the Botanical Survey of India for allowing us to use their facility to study and identify cyanobacteria based on morphology.

\section{REFERENCES}

1. Science - AlumierMD. Accessed May 19, 2021. https://beta. skinscience.alumiermd.com/skin-conditions/

2. Bhatia S, Sharma K, Namdeo AG, Chaugule BB, Kavale M, Nanda S. Broad-spectrum sun-protective action of Porphyra-334 derived from Porphyra vietnamensis. Pharmacognosy research. 2010 Jan;2(1):45.doi:10.4103/0974-8490.60578

3. Ratnasooriya WD, Abeysekera WPKM, Ratnasooriya CTD. In vitro anti-hyaluronidase activity of Sri Lankan low grown orthodox orange pekoe grade black tea (Camellia sinensis L.). Asian Pac J Trop Biomed. 2014;4(12):959-963. doi:10.12980/ APJTB.4.2014APJTB-2014-0462

4. Gupta PL, Rajput M, Oza T, Trivedi U, Sanghvi G. Eminence of Microbial Products in Cosmetic Industry. Nat Products Bioprospect. 2019;9(4):267-278. doi:10.1007/s13659-019-0215-0

5. Pignolet O, Jubeau S, Vaca-Garcia C, Michaud P. Highly valuable microalgae: Biochemical and topological aspects. J Ind Microbiol Biotechnol. 2013;40(8):781-796. doi:10.1007/s10295013-1281-7

6. Sigamani S, Ramamurthy D, Natarajan H. A review on potential biotechnological applications of microalgae. J Appl Pharm Sci. 2016;6(8):179-184. doi:10.7324/JAPS.2016.60829

7. Abd El-Aty AM, Mohamed AA, Samhan FA. In-vitro antioxidant and antibacterial activities of two fresh water Cyanobacterial species, Oscillatoria agardhii and Anabaena sphaerica. J Appl Pharm Sci. 2014;4(07):69-075. doi:10.7324/JAPS.2014. 40712

8. Wanigatunge RP, Magana-Arachchi DN, Chandrasekharan N V., Kulasooriya SA. Genetic diversity and molecular phylogeny of cyanobacteria from Sri Lanka based on 16S rRNA gene. Environ Eng Res. 2014;19(4):317-329. doi:10.4491/eer.2014.035

9. Ratnaparkhe S, Devyani Mali M, Bela Nabar M. Evaluation of free radical scavenging activity and skin whitening potential of cyanobacteria. Int J Adv Res Ideas Innov Technol. 2021;7(3). doi:xx.xxx/ijariit-v7i3-1250

10. Van Wychen S, Laurens LM. Determination of total carbohydrates in algal biomass: laboratory analytical procedure (LAP). National Renewable Energy Lab.(NREL), Golden, CO (United States); 2016 Jan 13. www.nrel.gov/publications. 
11. Byreddy AR, Gupta A, Barrow CJ, Puri M. A quick colorimetric method for total lipid quantification in microalgae. J Microbiol Methods. 2016;125:28-32. doi:10.1016/j.mimet.2016.04.002

12. Rastogi RP, Incharoensakdi A. Characterization of UVscreening compounds, mycosporine-like amino acids, and scytonemin in the cyanobacterium Lyngbya sp. CU2555. doi:10.1111/1574-6941.12220

13. Pathak J, Sonker AS, Kannaujiya VK, Sinha RP. Isolation and partial purification of scytonemin and mycosporinelike amino acids from biological crusts. J Chem Pharm Res. 2015;7(1):362-371

14. Shalaby E, Shanab SMM, Shalaby EA. Comparison of DPPH and ABTS Assays for Determining Antioxidant Potential of Water and Methanol Extracts of Spirulina Platensis. Vol 42.; 2013. https:// www.researchgate.net/publication/287536387

15. Jeffrey SW, MacTavish HS, Dunlap WC, Vesk M, Groenewoud K. Occurrence of UVA- and UVB-absorbing compounds in 152 species (206 strains) of marine microalgae. Mar Ecol Prog Ser. Published online November 26, 1999:35-51.

16. Ratnaparkhe S, Nabar Bela. Screening of algae for its antibacterial and antiacne potential. Indian J Appl Res. 2020;10(2):74-76. doi:10.36106/ijar

17. Cui HX, Duan FF, Jia SS, Cheng FR, Yuan K. Antioxidant and tyrosinase inhibitory activities of seed oils from torreya grandis Fort. ex Lindl. Biomed Res Int. 2018;2018. doi:10.1155/2018/5314320

18. Sari DM, Anwar E, Nurjanah, Arifianti AE. Antioxidant and tyrosinase inhibitor activities of ethanol extracts of brown seaweed (Turbinaria conoides) as lightening ingredient. Pharmacogn J. 2019;11(2):379-382. doi:10.5530/pj.2019.11.58

19. Gharbi K, Fathalli A, Fassatoui C, Romdhane MS, Jenhani ABR. Biochemical analysis of cyanobacterial strains isolated from different Tunisian inland waters: therapeutical and nutritional potential. Int J Biol Chem Sci. 2019;13(4):2286. doi:10.4314/ijbcs.v13i4.32

20. FAY F, Obando Claudia Z, Isabelle L, et al. Fatty Acid Profiling of Tropical Microalgae and Cyanobacteria Strains Isolated From Southwest Indian Ocean Islands. J Mar Biol Aquac. 2017;3(2):1-14. doi:10.15436/2381-0750.17.1367

21. Singh P, Kumar D. Biomass and Lipid Productivities of Cyanobacteria- Leptolyngbya foveolarum HNBGU001. BioEnergy Res. 2021;14(1):278-291. doi:10.1007/s12155-020-10170-3

22. Lawrence KP, Long PF, Young AR. Mycosporine-Like Amino Acids for Skin Photoprotection. Curr Med Chem. 2017;25(40):5512-5527. doi:10.2174/0929867324666170529124237

23. Pant A, Pandey R. Bioactive phytomolecules and aging in Caenorhabditis elegans. Heal Aging Res. Published online 2015. doi:10.12715/har.2015.4.19.

How to cite this article: : Ratnaparkhe S, Mali D, Nabar B. Study of potential application of Cyanobacteria Leptolyngbya Valderiana in skin cosmeceuticals. Int J Appl Pharm Sci Res. 2021;6(4):44-49. doi: https://doi.org/10.21477/ijapsr.6.4.01

Source of Support: Nil.

Conflict of Support: None declared. 